\title{
Day-90 survival in critically-ill patients with COVID-19 and hydroxychloroquine: a propensity analysis
}

\author{
Martin Cour ${ }^{1}$, Camille Amaz ${ }^{2}$ Julien Bohé ${ }^{3}$, Thomas Rimmelé $^{4}$, Michel Ovize $^{2}$, Laurent Argaud $^{1 \wedge}$ \\ ${ }^{1}$ Medical Intensive Care Department, Hospices Civils de Lyon, Edouard Herriot Hospital, Lyon, France; ${ }^{2}$ Hospices Civils de Lyon, Investigation \\ Clinical Center of Lyon, Lyon, France; ${ }^{3}$ Medical Intensive Care Department, Hospices Civils de Lyon, Lyon-Sud Hospital, Lyon, France; \\ ${ }^{4}$ Anesthesia and Critical Care Department, Hospices Civils de Lyon, Edouard Herriot Hospital, Lyon, France \\ Contributions: (I) Conception and design: L Argaud, M Cour; (II) Administrative support: L Argaud; (III) Provision of study materials or patients: \\ M Cour, J Bohé, T Rimmelé, L Argaud; (IV) Collection and assembly of data: M Cour, L Argaud; (V) Data analysis and interpretation: M Cour, C \\ Amaz, L Argaud; (VI) Manuscript writing: All authors; (VII) Final approval of manuscript: All authors. \\ Correspondence to: Prof. Laurent Argaud, MD, PhD. Service de Médecine Intensive-Réanimation, Hôpital Edouard Herriot, 5, Place d'Arsonval, \\ 69437 Lyon Cedex 03, France. Email: laurent.argaud@chu-lyon.fr.
}

Background: There are limited data on the effect of hydroxychloroquine on medium term outcomes in patients with coronavirus disease 2019 (COVID-19) requiring intensive care. We aimed to evaluate the effects of hydroxychloroquine on day 90 mortality in this specific population.

Methods: This retrospective, multicenter, propensity matched cohort analysis, used data of adult patients with laboratory confirmed COVID-19 admitted to 3 university affiliated intensive care units between March 7, 2020, to April 7, 2020 in Lyon, France. Patients received either hydroxychloroquine (loading dose of 400 $\mathrm{mg}$ twice daily at day 1 followed by $200 \mathrm{mg}$ twice daily from day 2 to day 10) or standard of care without hydroxychloroquine. We compared all-cause mortality at day-90 after ICU admission between propensity score matched groups receiving hydroxychloroquine or standard of care.

Results: A total of 157 patients were included with a day-28 and day-90 mortality rate of $23.6 \%$ and $32.5 \%$, respectively. The median (interquartile) age was 67 years (56-76 years), 105 (66.9\%) were men, 65 (41.4\%) fulfilled criteria for acute respiratory distress syndrome, and 64 (41\%) received hydroxychloroquine (HCQ) for 10 days (4-10 days). In the propensity score matched cohort (59 patients in each group), day-90 mortality was $35.6 \%$ for patients who received HCQ and $23.7 \%$ for patients who did not (P=0.23). Kaplan Meier survival analysis showed no statistically significant association between HCQ therapy and mortality ( $\mathrm{P}=0.20$ by log-rank test).

Conclusions: In this study, off-label use of HCQ in critically ill patients with COVID-19 was not associated with any significant change in medium-term prognosis, confirming results of studies in less severe patients.

Keywords: Coronavirus disease 2019 (COVID-19); severe acute respiratory syndrome coronavirus 2 (SARS$\mathrm{CoV}-2)$; hydroxychloroquine (HCQ); acute respiratory distress syndrome; propensity score

Submitted Dec 03, 2020. Accepted for publication Jan 12, 2021.

doi: $10.21037 / \mathrm{atm}-20-7811$

View this article at: http://dx.doi.org/10.21037/atm-20-7811

^ ORCID: 0000-0002-4565-2454. 


\section{Introduction}

The coronavirus disease 2019 (COVID-19) pandemic caused by severe acute respiratory syndrome coronavirus 2 (SARS$\mathrm{CoV}-2)$ is an ongoing global health crisis responsible for more than one million deaths (1). Very quickly after the first cases were observed, off-label use of hydroxychloroquine (HCQ) has been proposed for its potential antiviral/immunomodulatory properties to treat patients with COVID-19 infection (2). Preliminary reports had suggested clinical benefits for patients with mild COVID-19 symptoms, without major safety concerns $(3,4)$. This led many physicians to also consider HCQ for patients requiring intensive care for acute respiratory failure, i.e., those who might most benefit from effective drugs with both antiviral and anti inflammatory therapy (5). As of May 2020, based on the neutral results of both observational studies and trials assessing whether HCQ would improve short-term outcomes (i.e., <30 days) in patients with mildto-moderate COVID-19 (6-10), many national public health agencies have recommended not to use HCQ to treat the disease. In November 2020, a large trial reported no short-term benefits of this treatment, including in the subgroup of patients receiving invasive mechanical ventilation (11). However, there is still a lack of data supporting or disproving off-label use of HCQ in the specific population of critically ill patients, while safety concerns have been reported $(5,12)$. Moreover, to our knowledge, no study has evaluated the potential benefits/ harms of HCQ on medium-term outcomes. This is all the more important as a high proportion of critically ill COVID-19 patients remain hospitalized in ICU or even mechanically one month after admission with still a risk of poor outcome.

Therefore, we conducted a propensity-matched cohort analysis to determine the effect of HCQ on day-90 mortality of patients with COVID-19 admitted to ICU as compared to standard of care.

We present the following article in accordance with the STROBE reporting checklist (13) (available at http://dx.doi. org/10.21037/atm-20-7811).

\section{Methods}

This study was approved by our institutional ethics committee (Comité d'Ethique du CHU de Lyon), approval protocol number $n^{\circ} 20-42$, with a waiver for written inform consent because of the retrospective nature of the study. All procedures performed in this study were in accordance with the ethical standards of our institutional research committee and with the 1964 Declaration of Helsinki and its later amendments. The methodology of this study was consistent with the STROBE statement for observational studies (13).

\section{Study population}

We retrospectively reviewed all adult patients with laboratory-confirmed COVID-19, admitted from 7 March 2020 to 7 April 2020, to 3 university-affiliated ICUs in Lyon, France. Those who stayed at least 24 hours in ICU and who received HCQ or no antiviral against SARS CoV-2 were included in this study. Follow-up continued until 5 July 2020. HCQ (loading dose of $400 \mathrm{mg}$ twice daily at day 1 followed by $200 \mathrm{mg}$ twice daily from day 2 to day 10) was prescribed at the discretion of the physician in charge, in accordance to national interim guidance on the management of critically ill patients with COVID-19 (14).

\section{Data collection}

For each patient, demographics, comorbidities, time from onset of COVID-19 symptoms to ICU admission, initial presentation of the disease (within 24 hours after ICU admission) including presence of acute respiratory distress syndrome (ARDS) in patients receiving invasive mechanical ventilation according to the Berlin criteria (15), Sequential Organ Failure Assessment score (16) (range, 0-24, with higher scores indicating more severe organ failures) calculated using the worst clinical and/or biological values from ICU admission to 24 hours later, organ dysfunctions as defined by a SOFA sub-score $\geq 1$, and Simplified Acute Physiology Score II (17) (SAPS II; range, 0-164, with higher scores indicating greater severity of illness) were documented. Severity of hypoxemia at ICU admission was estimated with the ratio of the arterial partial pressure of oxygen to the fractional inspired oxygen $\left(\mathrm{PaO}_{2} / \mathrm{FiO}_{2}\right)$; in case of missing data (i.e., when arterial blood gas was not performed), the $\mathrm{PaO}_{2} / \mathrm{FiO}_{2}$ ratio was imputed from pulse oximetry and $\mathrm{FiO}_{2}$, as previously described (18). Organ supports during the first 28 days after ICU admission were also collected.

\section{Outcomes}

The primary outcome was all-cause mortality at day-90 after ICU admission.

\section{Statistical analysis}

Continuous data are expressed as median (interquartile 


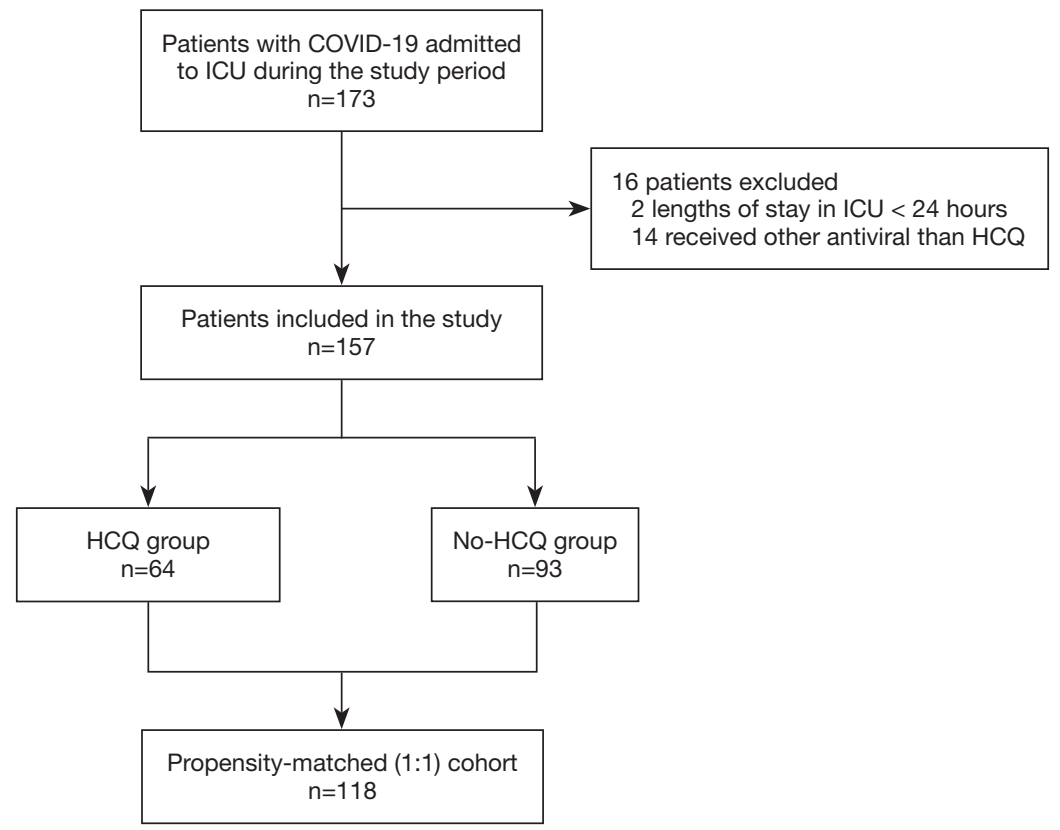

Figure 1 Study cohort. COVID-19, coronavirus disease 2019; ICU, intensive care unit; HCQ, hydroxychloroquine.

range) and qualitative data as number (proportion). Continuous and categorical variables were compared using Student's t test or Wilcoxon-Mann-Whitney test, and $\mathrm{Chi}^{2}$ or Fisher's exact test, as appropriate. Logistic regression analysis was used to determine baseline factors independently associated with day-90 mortality.

Since patients were not randomly assigned to receive HCQ, propensity score matching (19) was used to compare similar patient population receiving or not HCQ. The sample size was determined by the number of eligible patients admitted to ICU during the study period. We compared groups by intention-to-treat analysis, regardless of the discontinuation of HCQ during follow-up. There was no imputation for missing data. Covariates presumed to be associated with both the decision of HCQ therapy and outcomes or unbalanced between groups were used to build the propensity score (i.e., age, sex, chronic renal and cardiac diseases, ARDS, duration of symptoms before admission and SOFA score). The nearest neighbor method with a caliper of 0.2 was applied to create a matched cohort of HCQ-treated and untreated patients.

Survival curves among propensity-matched patients were constructed using Kaplan Meier estimates with comparison between curves based on the log-rank test.

All statistical analyses were performed using $\mathrm{R}$ (version 3.3.3, R Foundation for Statistical Computing) software.
Statistical significance was considered at 2 -sided $\mathrm{P}<0.05$.

\section{Results}

Of 173 patients with COVID-19 who were admitted to ICUs during the study period, 16 (9.2\%) were excluded because ICU length of stay was less than 24 hours or because they received antivirals other than HCQ or HCQ before ICU admission (Figure 1). Thus, 157 patients [median age 67 years (interquartile range, 56-76 years); 105 men (66.8\%)] were included in the analysis with no loss in follow-up. At admission, all patients presented with COVID-19-induced acute respiratory symptoms, including measured or imputed $\mathrm{PaO}_{2} / \mathrm{FiO}_{2}$ ratios $<400 \mathrm{mmHg}$, and required oxygen supplementation. A large majority of them $(125 / 157,80 \%)$ were severely hypoxemic with $\mathrm{PaO}_{2} / \mathrm{FiO}_{2}$ ratio $<200 \mathrm{mmHg}$. Among the 157 patients, 64 (40.8\%) received HCQ for 10 days (4-10 days). HCQ treatment was prematurely stopped after 4 days (3-6 days) for 28 (43.8\%) patients due to acute renal failure in $13(20.3 \%)$ cases, QT interval prolongation in $10(15.6 \%)$ cases or for preventing drug drug interaction in $5(7.8 \%)$ cases. No long QT induced arrhythmia was recorded in HCQ treated patients.

Before matching, patients receiving HCQ were significantly younger, had less chronic heart disease, and lower SAPS II than patients not treated with HCQ (Table 1). Mortality at day 90 
Table 1 Patients' characteristics and outcomes before and after matching on the propensity score for hydroxychloroquine therapy

\begin{tabular}{|c|c|c|c|c|c|c|}
\hline Variables & \multicolumn{3}{|c|}{ Before matching } & \multicolumn{3}{|c|}{ After matching } \\
\hline \multicolumn{7}{|l|}{ Demographics } \\
\hline Age (years) & $64[53-70]$ & $72[61-79]$ & 0.001 & $65[53-72]$ & $65[56-73]$ & 0.457 \\
\hline Male sex, n (\%) & $47(73.4)$ & $58(62.4)$ & 0.202 & $43(72.9)$ & $42(71.2)$ & 0.999 \\
\hline \multicolumn{7}{|l|}{ Comorbidities, n (\%) } \\
\hline Hypertension & $29(45.3)$ & $51(54.8)$ & 0.312 & $28(47.5)$ & $29(49.2)$ & 0.999 \\
\hline Diabetes & $16(25.0)$ & $28(30.1)$ & 0.603 & $14(23.7)$ & $18(30.5)$ & 0.534 \\
\hline Chronic renal diseases & 7 (10.9) & $14(15.1)$ & 0.613 & 7 (11.9) & $6(10.2)$ & 0.999 \\
\hline Simplified Acute Physiology Score II (points) & 33 [25-37] & 35 [29-47] & 0.044 & 35 [26-38] & 32 [25-44] & 0.974 \\
\hline \multicolumn{7}{|l|}{ Initial presentation } \\
\hline ARDS, n (\%) & $28(43.8)$ & $37(39.8)$ & 0.741 & $26(44.1)$ & $26(44.1)$ & 0.999 \\
\hline Severity of ARDS, n (\%) & & & 0.116 & & & 0.071 \\
\hline Mild & $0(0.0)$ & $3(8.1)$ & & $0(0.0)$ & $3(11.5)$ & \\
\hline Moderate & $15(53.6)$ & $24(64.9)$ & & $14(53.8)$ & $17(65.4)$ & \\
\hline Severe & $13(46.4)$ & $10(27.0)$ & & $12(46.2)$ & $6(23.1)$ & \\
\hline Lowest $\mathrm{PaO}_{2} / \mathrm{FiO}_{2}(\mathrm{mmHg})^{\dagger}$ & $142[82-215]$ & $141[98-186]$ & 0.661 & 142 [82-228] & $143[98-180]$ & 0.580 \\
\hline Hematological & $13(20.3)$ & $13(14.0)$ & 0.406 & $12(20.3)$ & $9(15.3)$ & 0.630 \\
\hline Renal & $13(20.3)$ & $28(30.1)$ & 0.235 & $12(22.0)$ & $11(18.6)$ & 0.819 \\
\hline Hepatic & $5(7.8)$ & $6(6.5)$ & 0.992 & $4(6.8)$ & $4(6.8)$ & 0.999 \\
\hline Number & $2[1-3]$ & $2[2-3]$ & 0.229 & $2[1-3]$ & $2[1.5-3]$ & 0.805 \\
\hline SOFA score (points) & $3[2-7]$ & $3[3-7]$ & 0.279 & $3[2-7]$ & $3[2-6]$ & 0.883 \\
\hline \multicolumn{7}{|l|}{ Organ support, n (\%) } \\
\hline Invasive mechanical ventilation & $37(57.8)$ & $46(49.5)$ & 0.386 & $35(59.3)$ & $32(54.2)$ & 0.710 \\
\hline Vasoactive drugs & $35(54.7)$ & $45(48.4)$ & 0.540 & $33(55.9)$ & $31(52.5)$ & 0.853 \\
\hline Renal replacement therapy & $16(25.0)$ & $17(18.3)$ & 0.414 & $15(25.4)$ & $12(20.3)$ & 0.661 \\
\hline
\end{tabular}

Table 1 (continued) 
Table 1 (continued)

\begin{tabular}{|c|c|c|c|c|c|c|}
\hline Variables & \multicolumn{3}{|c|}{ Before matching } & \multicolumn{3}{|c|}{ After matching } \\
\hline \multicolumn{7}{|l|}{ Outcomes } \\
\hline Day-28 mortality, n (\%) & $15(23.4)$ & $22(23.7)$ & 0.999 & $13(22.0)$ & $10(16.9)$ & 0.642 \\
\hline Day-28 ventilation free days & 6.5 [2-28] & 16 [3-28] & 0.396 & 5 [2-28] & 13 [3-28] & 0.357 \\
\hline
\end{tabular}

Continuous values are expressed median (interquartile range). ${ }^{\dagger}, 5$ values were missing (2 in the HCQ group and 3 in the No-HCQ group) within the first 48 hours; ${ }^{\ddagger}$, Organ dysfunction was defined as a SOFA sub-score $\geq 1$. HCQ, hydroxychloroquine; ICU, intensive care unit; ARDS, acute respiratory distress syndrome; $\mathrm{PaO}_{2} / \mathrm{FiO}_{2}$, ratio of the arterial partial pressure of oxygen to the fractional inspired oxygen; SOFA, sequential organ failure assessment.

did not significantly differ between the 2 unmatched groups (35.9\% in the HCQ group versus $30.1 \%$ in the No-HCQ group; $\mathrm{P}=0.553)$. Age, duration of symptoms before admission, SAPS II, ARDS, lowest $\mathrm{PaO}_{2} / \mathrm{FiO}_{2}$ ratio and renal failure within the first 24 hours after ICU admission, SOFA score at 24 hours after ICU admission and organ supports during ICU stay were significantly associated with day 90 mortality (Table 2). In multivariate analysis, age and SOFA score at day 1 were independently $(\mathrm{P}<0.01)$ associated with day-90 mortality (Table 2).

Propensity score matching patients treated with HCQ or not yielded 59 matched pairs with well-balanced baseline characteristics (Table 1 and Figure 2). In this matched cohort, day-28 mortality and day-90 mortality were $22.0 \%$ and $35.6 \%$, respectively, for patients who received HCQ versus $16.9 \%$ and $23.7 \%$ for patients who did not (Table 1). As reported in Figure 3, Kaplan-Meier survival analysis before and after matching showed no statistically significant association between HCQ therapy and mortality.

\section{Discussion}

This report presents new data regarding day-90 mortality of critically ill patients with COVID-19 and on the efficacy of HCQ for treating this specific population. In this study involving patients with COVID-19 requiring intensive care, off-label use of HCQ was not associated with any significant change in day-90 mortality as compared to standard of care, even after matching patients with similar characteristics.

To our knowledge, no previous study had specifically examined the effects of HCQ on clinically relevant medium-term outcomes in patients with the most severe presentations of COVID-19. The absence of benefits of HCQ reported herein is in line with previous well designed observational studies and randomized trials showing no improvement in survival in non critically ill patients receiving HCQ (6-10). The efficacy and safety of HCQ in the most severe patients who often require mechanical ventilation and receive multiple co medications but also benefit from close monitoring could not be extrapolated from these studies. Our study population comprising critically ill patients with a median age over 60 years with major comorbidities requiring organ support in more than half of cases, was quite similar to other unselected ICU cohorts in western countries (20-23). Propensity matching was unable to detect any benefit of HCQ on the day-90 mortality rate. It is difficult to compare the mortality observed in this work to other studies because data on medium-term survival in patients with COVID 19 are scarce. In most observational studies or randomized trials, the follow up did not exceed 28 days $(6-11,20-23)$. At this timepoint, mortality in both groups was more than $10 \%$ lower than the one reported in the RECOVERY trial (11). Interestingly, we found that mortality increased by about $30 \%$ between day 28 and day 90 . We should therefore be cautious when interpreting the short-term results of studies investigating drugs or intervention with the potential of modifying mortality in this specific population.

We did not record any excess of life threatening arrhythmia in HCQ-treated patients. This might partly be due to the close monitoring of drug-related cardiac adverse events in ICU and to the fact that HCQ was stopped preemptively for $43.8 \%$ of patients, mostly because of prolonged QT interval and renal failure. Nevertheless, high proportion of patients who could not receive the complete 
Table 2 Patient characteristics according to day- 90 mortality

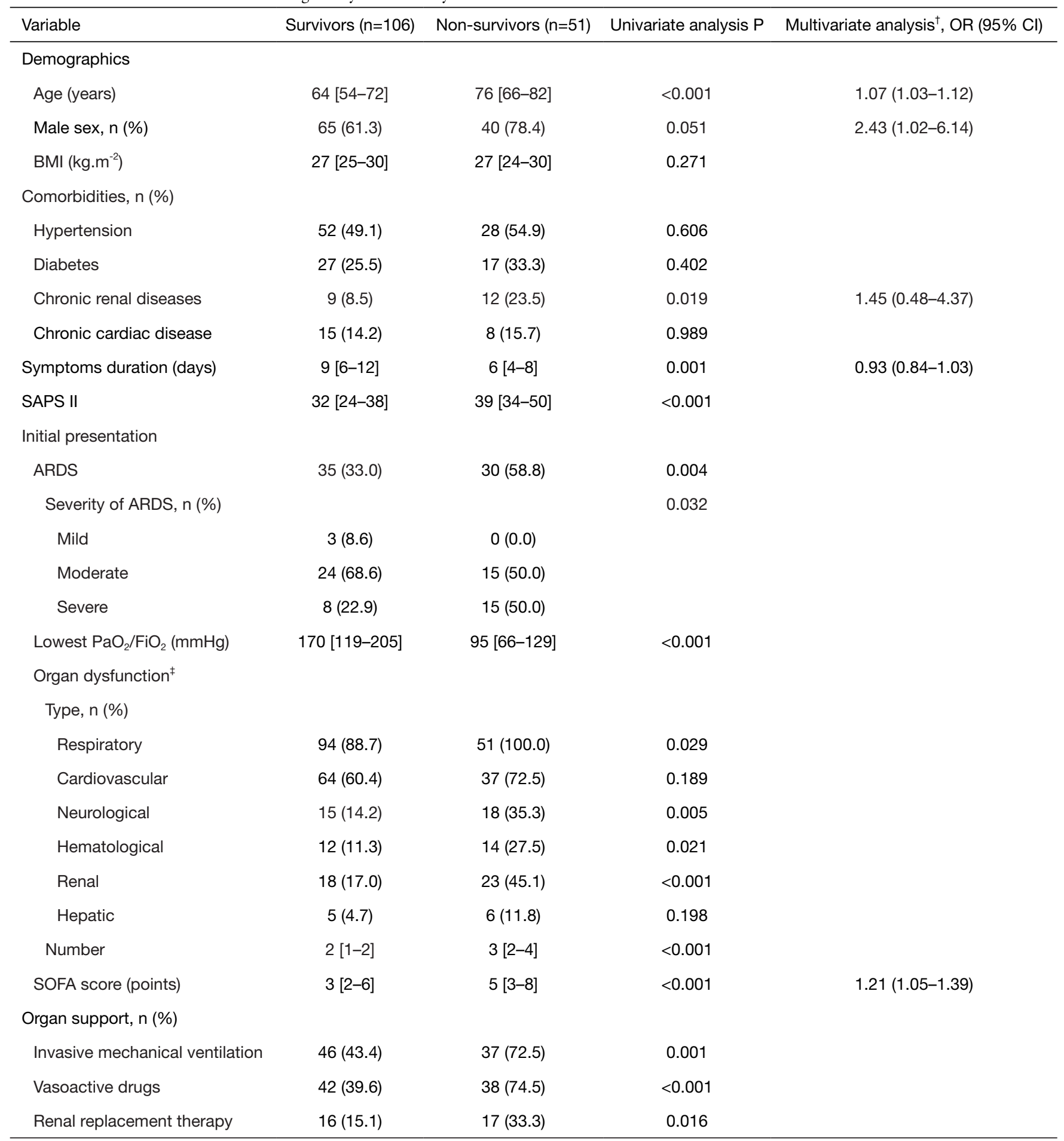

${ }^{\dagger}$, SAPSII, ARDS, lowest $\mathrm{PaO}_{2} / \mathrm{FiO}_{2}$ and organ dysfunctions were not entered into the model because of collinearity with the SOFA score. $\ddagger$, Organ dysfunction was defined as a SOFA sub-score $\geq 1$. Continuous values are expressed median (interquartile range). OR, odds ratio; Cl, confidence intervals; BMI, body mass index; ICU, intensive care unit; SAPS II, simplified acute physiology score II; ARDS, acute respiratory distress syndrome; $\mathrm{PaO}_{2} / \mathrm{FiO}_{2}$, ratio of the arterial partial pressure of oxygen to the fractional inspired oxygen; SOFA, sequential organ failure assessment. 

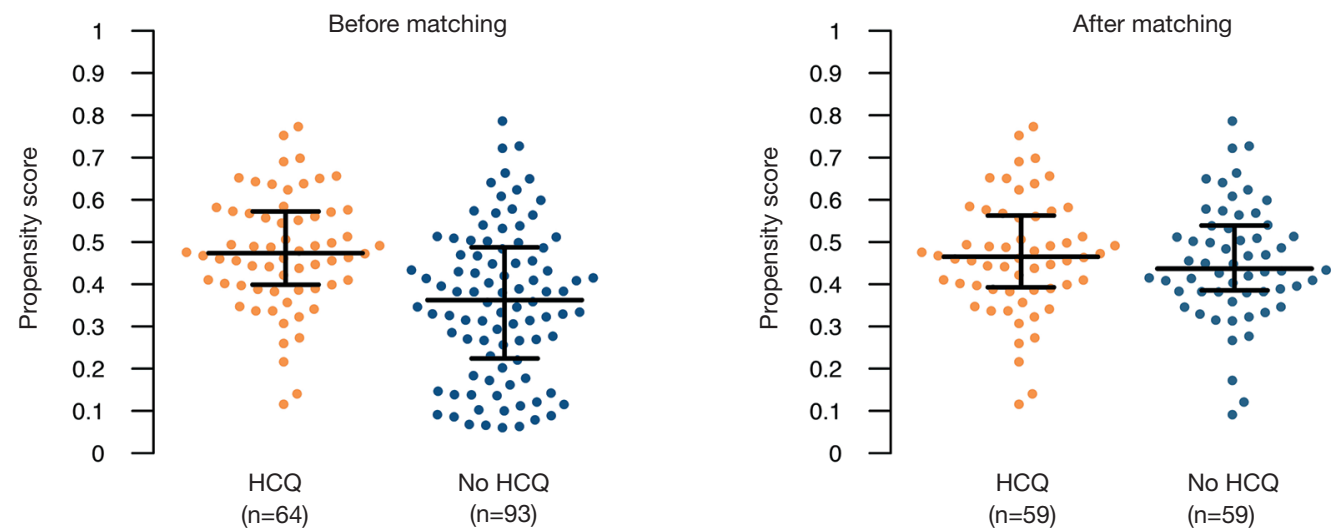

Figure 2 Individual propensity scores before and after matching. Individual propensity score values are shown for patients with COVID-19 treated with HCQ or No-HCQ before (left panel) and after (right panel) matching. Median and interquartile range of propensity scores are also presented. COVID-19, coronavirus disease 2019; HCQ, hydroxychloroquine.

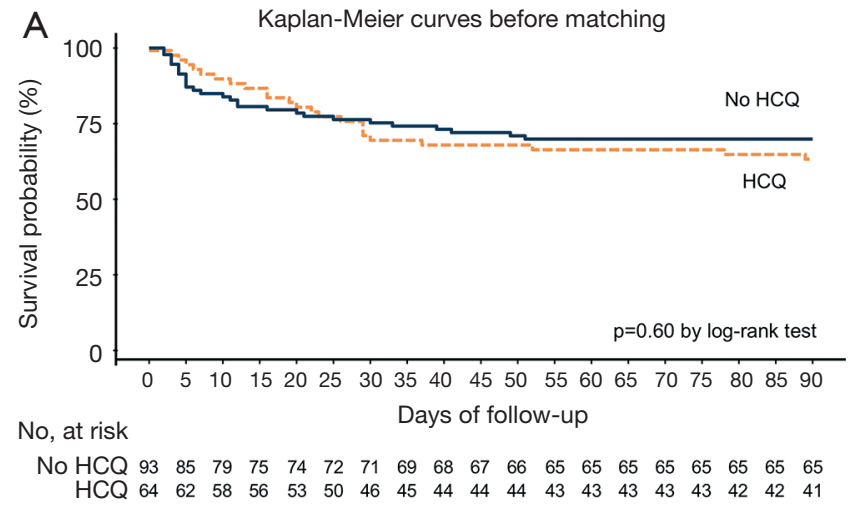

B

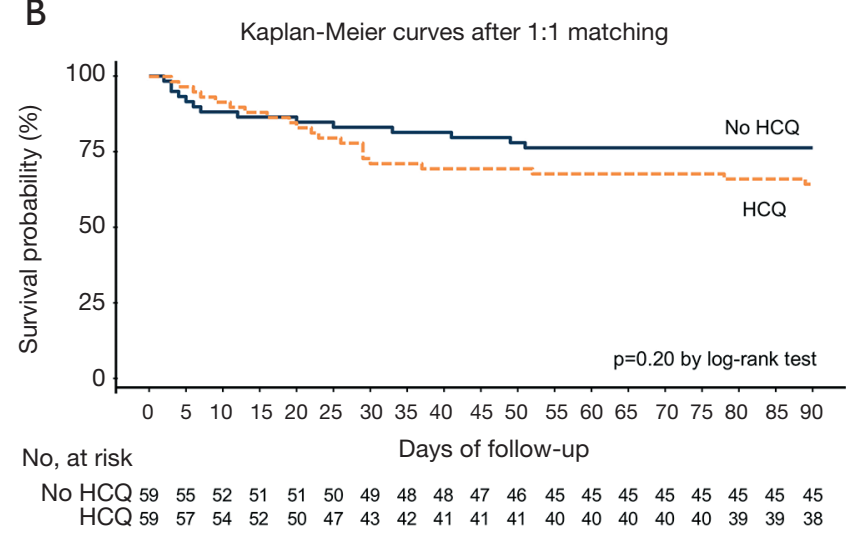

Figure 3 Kaplan-Meier survival curves of propensity score matched patients with COVID 19 treated or not with hydroxychloroquine. Kaplan-Meier curves are presented before (panel A) and after (panel B) matching patients receiving HCQ or standard of care (No HCQ). COVID-19, coronavirus disease 2019; HCQ, hydroxychloroquine. treatment highlights difficulties in managing this therapy in ICU where contraindications and warning of use (e.g., acute renal failure) or the need for other therapy prolonging the QT interval, are frequent.

The study has several limitations. First, although we used propensity score matching to ensure comparability between study groups, potential unmeasured confounders could have biased our conclusion. Second, the small sample size could have led to underpowered analysis and did not allow subgroup analysis. Third, we cannot rule out that other $\mathrm{HCQ}$ regimen could have provided different results. Nevertheless, we used dose of HCQ and duration that have been determined as optimal for treating hospitalized patients with COVID-19 (24). Yet, the fact that HCQ had to be stopped prematurely in half of patients might limit the conclusions about its efficacy and/ or toxicity in critically-ill patients. Fourth, the study was conducted in 3 French academic ICUs, which may limit its generalizability to other settings or countries, even if characteristics of our cohort were comparable to other cohorts from western countries (20-23).

\section{Conclusions}

In summary, among critically ill patients with COVID-19, off-label use of HCQ was not associated with any significant change in day-90 mortality when compared with standard of care, after matching patients to account for baseline differences. Our results suggest that HCQ should not be used to treat the most severe forms of the disease. 


\section{Acknowledgments}

The authors would like to thank Marion Proven and Sylvie de la Salle for essential assistance in conducting the study. We also thank for his technical support the framework RHU MARVELOUS (ANR 16 RHUS 0009) of the Université Claude Bernard Lyon 1 (UCBL), within the program "Investissement d'Avenir" operated by the French National Research Agency.

Funding: None.

\section{Footnote}

Reporting Checklist: Available at http://dx.doi.org/10.21037/ atm-20-7811

Data Sharing Statement: Available at http://dx.doi. org/10.21037/atm-20-7811

Conflicts of Interest: All authors have completed the ICMJE uniform disclosure form (available at http://dx.doi. org/10.21037/atm-20-7811). The authors have no conflicts of interest to declare.

Ethical Statement: The authors are accountable for all aspects of the work in ensuring that questions related to the accuracy or integrity of any part of the work are appropriately investigated and resolved. The study was conducted in accordance with the Declaration of Helsinki (as revised in 2013). The study was approved the institutional ethics committee of the CHU de Lyon, approval protocol number $n^{\circ} 20-42$, with a waiver for written inform consent because of the retrospective nature of the study.

Open Access Statement: This is an Open Access article distributed in accordance with the Creative Commons Attribution-NonCommercial-NoDerivs 4.0 International License (CC BY-NC-ND 4.0), which permits the noncommercial replication and distribution of the article with the strict proviso that no changes or edits are made and the original work is properly cited (including links to both the formal publication through the relevant DOI and the license). See: https://creativecommons.org/licenses/by-nc-nd/4.0/.

\section{References}

1. World Health Organization. Coronavirus disease (COVID-2019) situation report. Date last accessed:
November 26, 2020. Available online: https://www. who.int/emergencies/diseases/novel-coronavirus-2019/ situation-reports

2. Yao X, Ye F, Zhang M, et al. In Vitro Antiviral Activity and Projection of Optimized Dosing Design of Hydroxychloroquine for the Treatment of Severe Acute Respiratory Syndrome Coronavirus 2 (SARS-CoV-2). Clin Infect Dis 2020;71:732-9.

3. Gao J, Tian Z, Yang X. Breakthrough: Chloroquine phosphate has shown apparent efficacy in treatment of COVID-19 associated pneumonia in clinical studies. Biosci Trends 2020;14:72-3.

4. Gautret P, Lagier JC, Parola P, et al. Hydroxychloroquine and azithromycin as a treatment of COVID-19: results of an open-label non-randomized clinical trial. Int J Antimicrob Agents 2020;56:105949.

5. Taccone FS, Gorham J, Vincent JL. Hydroxychloroquine in the management of critically ill patients with COVID-19: the need for an evidence base. Lancet Respir Med 2020;8:539-41.

6. Geleris J, Sun Y, Platt J, et al. Observational Study of Hydroxychloroquine in Hospitalized Patients with Covid-19. N Engl J Med 2020;382:2411-8.

7. Rosenberg ES, Dufort EM, Udo T, et al. Association of treatment with hydroxychloroquine or azithromycin with in-hospital mortality in patients with COVID-19 in New York state. JAMA 2020;323:2493-502.

8. Mahévas M, Tran VT, Roumier M, et al. Clinical efficacy of hydroxychloroquine in patients with covid-19 pneumonia who require oxygen: observational comparative study using routine care data. BMJ 2020;369:m1844.

9. Tang W, Cao Z, Han M, et al. Hydroxychloroquine in patients with mainly mild to moderate coronavirus disease 2019: open label, randomised controlled trial. BMJ 2020;369:m1849.

10. Cavalcanti AB, Zampieri FG, Rosa RG, et al. Hydroxychloroquine with or without Azithromycin in Mild-to-Moderate Covid-19. N Engl J Med 2020;383:2041-52.

11. RECOVERY Collaborative Group, Horby P, Mafham $M$, et al. Effect of Hydroxychloroquine in Hospitalized Patients with Covid-19. N Engl J Med 2020;383:2030-40.

12. Bessière $\mathrm{F}$, Roccia $\mathrm{H}$, Delinière $\mathrm{A}$, et al. Assessment of QT Intervals in a Case Series of Patients With Coronavirus Disease 2019 (COVID-19) Infection Treated With Hydroxychloroquine Alone or in Combination With Azithromycin in an Intensive Care Unit. JAMA Cardiol 2020;5:1067-9. 
13. von Elm E, Altman DG, Egger M, et al. The Strengthening the Reporting of Observational Studies in Epidemiology (STROBE) Statement: Guidelines for Reporting Observational Studies. PLoS Med 2007;4:e296

14. SRLF, SFAR, SFMU, GFRUP, SPILF, SPLF (2020)

Recommandations d'experts portant sur la prise en charge en réanimation des patients en période d'épidémie à SARS CoV-2. Data last accessed March 1, 2020. Available online: https://www.srlf.org/wp-content/uploads/2020/04/RFECOVID_V4.pdf

15. Ferguson ND, Fan E, Camporota L, et al. The Berlin definition of ARDS: an expanded rationale, justification, and supplementary material. Intensive Care Med 2012;38:1573-82.

16. Ferreira FL, Bota DP, Bross A, et al. Serial evaluation of the SOFA score to predict outcome in critically ill patients. JAMA 2001;286:1754-8.

17. Le Gall JR, Loirat P, Alperovitch A, et al. A simplified acute physiology score for ICU patients. Crit Care Med 1984;12:975-7.

18. Gadrey SM, Lau CE, Clay R, et al. Imputation of partial pressures of arterial oxygen using oximetry and its impact

Cite this article as: Cour M, Amaz C, Bohé J, Rimmelé T, Ovize M, Argaud L. Day-90 survival in critically-ill patients with COVID-19 and hydroxychloroquine: a propensity analysis. Ann Transl Med 2021;9(7):524. doi: 10.21037/atm-20-7811 on sepsis diagnosis. Physiol Meas 2019;40:115008.

19. D'Agostino RB Jr. Propensity score methods for bias reduction in the comparison of a treatment to a nonrandomized control group. Stat Med 1998;17:2265-81.

20. Azoulay E, Zafrani L, Mirouse A, et al. Clinical phenotypes of critically ill COVID-19 patients. Intensive Care Med 2020;46:1651-2.

21. Piva S, Filippini M, Turla F, et al. Clinical presentation and initial management critically ill patients with severe acute respiratory syndrome coronavirus 2 (SARS-CoV-2) infection in Brescia, Italy. J Crit Care 2020;58:29-33.

22. Mitra AR, Fergusson NA, Lloyd-Smith E, et al. Baseline characteristics and outcomes of patients with COVID-19 admitted to intensive care units in Vancouver, Canada: a case series. CMAJ 2020;192:E694-701.

23. Bhatraju PK, Ghassemieh BJ, Nichols M, et al. Covid-19 in critically ill patients in the Seattle region - case series. $\mathrm{N}$ Engl J Med 2020;382:2012-22.

24. Lê MP, Peiffer-Smadja N, Guedj J, et al. Rationale of a loading dose initiation for hydroxychloroquine treatment in COVID-19 infection in the DisCoVeRy trial. J Antimicrob Chemother 2020;75:2376-80. 